\title{
Double carbapenem as a rescue strategy for the treatment of severe carbapenemase-producing Klebsiella pneumoniae infections: a two-center, matched case-control study
}

Gennaro De Pascale ${ }^{1,6^{*}}$, Gennaro Martucci ${ }^{2}$, Luca Montini', Giovanna Panarello², Salvatore Lucio Cutuli ${ }^{1}$, Daniele Di Carlo ${ }^{3}$, Valentina Di Gravio ${ }^{1}$, Roberta Di Stefano ${ }^{4}$, Guido Capitanio², Maria Sole Vallecoccia', Piera Polidori ${ }^{4}$, Teresa Spanu ${ }^{5}$, Antonio Arcadipane ${ }^{2}$ and Massimo Antonelli ${ }^{1}$

\begin{abstract}
Background: Recent reports have suggested the efficacy of a double carbapenem (DC) combination, including ertapenem, for the treatment of carbapenem-resistant Klebsiella pneumoniae (CR-Kp) infections. We aimed to evaluate the clinical impact of such a regimen in critically ill patients.
\end{abstract}

Methods: This case-control (1:2), observational, two-center study involved critically ill adults with a microbiologically documented CR-Kp invasive infection treated with the DC regimen matched with those receiving a standard treatment (ST) (i.e., colistin, tigecycline, or gentamicin).

Results: The primary end point was 28-day mortality. Secondary outcomes were clinical cure, microbiological eradication, duration of mechanical ventilation and of vasopressors, and 90-day mortality. Forty-eight patients treated with DC were matched with 96 controls. Occurrence of septic shock at infection and high procalcitonin levels were significantly more frequent in patients receiving DC treatment $(p<0.01)$. The 28 -day mortality was significantly higher in patients receiving ST compared with the DC group (47.9\% vs $29.2 \%, p=0.04)$. Similarly, clinical cure and microbiological eradication were significantly higher when DC was used in patients infected with CR-Kp strains resistant to colistin (13/20 (65\%) vs 10/32 (31.3\%), $p=0.03$ and $11 / 19(57.9 \%)$ vs $7 / 27$ (25.9\%), $p=0.04$, respectively). In the logistic regression and multivariate Cox-regression models, the DC regimen was associated with a reduction in 28 -day mortality (OR $0.33,95 \% \mathrm{Cl} 0.13-0.87$ and OR $0.43,95 \% \mathrm{Cl} 0.23-0.79$, respectively).

Conclusions: Improved 28-day mortality was associated with the DC regimen compared with ST for severe CR-Kp infections. A randomized trial is needed to confirm these observational results.

Trial registration: ClinicalTrials.gov NCT03094494. Registered 28 March 2017.

Keywords: Double carbapenem, Klebsiella pneumoniae, Critically ill patients, Infections, Multidrug-resistant bacteria, Meropenem, Ertapenem

\footnotetext{
*Correspondence: gennaro.depascalemd@gmail.com

${ }^{1}$ Department of Anesthesiology and Intensive Care, Università Cattolica del

Sacro Cuore, Fondazione Policlinico Agostino Gemelli, Rome, Italy

${ }^{6}$ Fondazione Policlinico A. Gemelli. Università Cattolica del Sacro Cuore,

Largo A. Gemelli 8, 00168 Rome, Italy

Full list of author information is available at the end of the article
} 


\section{Background}

Over the last two decades, Klebsiella pneumoniae, one of the most common nosocomial pathogens, has accumulated many resistance mechanisms. After its first isolation in 1996, in the United States, carbapenem-resistant Klebsiella pneumonaiae (CR-Kp) strains have caused numerous outbreaks of nosocomial infections in the northeastern United States, Israel, Greece, and Italy, becoming a serious clinical issue in these areas [1-3].

$\mathrm{CR}-K p$ isolates are spreading worldwide in an ongoing dynamic process, constituting a new global health threat. Infections related to this pathogen are associated with increased mortality, longer hospital and intensive care unit (ICU) stay, and consequent increased cost of care [4, 5].

Hospitalized patients with several comorbidities, poor functional status, and prior exposure to antibiotics are typically affected with such infections [6]. Preventive strategies aimed at reducing the spread of CR-Kp in the ICU are essential in reducing the infection rate related to these bacteria, which are a serious challenge for ICU physicians due to the exiguous available therapeutic options [7-10]. CR$K p$ has an extensive drug resistance phenotype, and even the use of rescue antimicrobials (i.e., tigecycline, aminoglycosides, colistin, and fosfomycin) is frequently associated with clinical failure. Furthermore, although several reports suggest that the combination of more than one in-vitro active drug may be superior to monotherapy, the percentage of clinical failure remains high [11-14]. Conversely, among new antimicrobials, the use of novel beta-lactam/beta-lactamase inhibitor combinations (e.g., ceftazidime-avibactam) is still poor and is associated with the rapid emergence of resistance during treatment $[15,16]$.

Recently, the association of two synergistic carbapenems (ertapenem plus either meropenem, doripenem, or imipenem), alone or combined with other antibiotics, has been proposed as rescue treatment for CR-Kp infections [17-19].

Such combination therapy was prompted by evidence that ertapenem has the least in-vitro activity against CR$K p$ due to an increased affinity for carbapenemases. Its role, as a suicide antibiotic, would allow the most active carbapenems to express their stronger activity at the infection site. However, although the data are encouraging, they are limited to in-vitro studies, case reports, and short case series [20, 21].

In this two-center, matched case-control study we analyzed the clinical and microbiological outcomes of patients affected with severe CR-Kp infections treated with a double carbapenem (DC) regimen, including ertapenem, compared with a standard rescue antibiotic treatment (ST).

\section{Methods}

\section{Patient population and study design}

This study was conducted in the general ICUs of two tertiary hospitals in Italy admitting approximately 1500 patients per year. We performed a retrospective 1:2 matched case-control analysis on data prospectively acquired from electronic ICU charts and computerized investigation of microbiology laboratory databases. Because of its observational, noninterventional design, the study was approved by the local ethics committees, which waived informed consent (approval numbers: UCSC14669/15; EC Palermo $\left.2 \mathrm{n}^{\circ} 359 / 2015\right)$. Eligibility criteria were as follows: age $\geq 18$ years; ICU admission between November 2012 and December 2015; documented CR-Kp invasive infection (i.e., pneumonia, bloodstream infection, complicated intraabdominal, skin and soft tissue, and urinary tract infections); and targeted antibiotic therapy lasting $\geq 72$ hours. Patients who received DC treatment, including ertapenem, were matched with those treated with other standard antibiotic combinations (ST). Matching was based on severity of illness as defined by the Simplified Acute Physiology Score (SAPS) II at ICU admission (within 5 points), and by the Sequential Organ Failure Assessment (SOFA) scores (within 2 points) upon diagnosis of infection [22, 23]. When multiple control candidates met the core matching criteria, the choice was based on ICU admission dates. Investigators were blinded to case outcomes during matching.

\section{Treatment regimens}

Meropenem and ertapemen were administered every 8 hours and 12/24 hours, respectively, at a daily dose of $6 \mathrm{~g}$ and $2 \mathrm{~g}$, respectively, adopting the extended infusion strategy (at least 3 hours) [24]. Colistin was administered every 12 hours at a daily dose of 9 million international units (MIU), after a 9 MIU loading dose. Gentamicin was administered every 24 hours at a daily per-kilogram dose of $5 / 7 \mathrm{mg}$. Tigecycline was administered at $100 \mathrm{mg}$ every 12 hours after a $200 \mathrm{mg}$ loading dose. Dosages were not changed during continuous renal replacement therapy (CRRT), including the colistin total daily dose, and were reduced in the presence of renal failure (except for tigecycline). Aminoglycoside therapeutic drug monitoring (TDM) was performed routinely.

\section{Definitions and outcomes}

Ventilator-associated pneumonia (VAP), bloodstream infections (BSIs), intra-abdominal infections (IAIs), skin and soft tissue infections (SSTIs), urinary tract infections (UTIs), septic shock (SS) status, and acute respiratory distress syndrome (ARDS) were classified according to current guidelines [25-31]. The primary outcome endpoint was 28-day mortality; secondary outcomes were clinical cure, microbiological eradication, duration of mechanical ventilation and of vasopressors, and 90-day mortality. Clinical cure was defined as the complete resolution of all signs and symptoms of the infection by the end of targeted therapy. Improvement or lack of progression of all abnormalities on chest radiographs was 
also required for VAP. Microbiological eradication was defined as the absence of the original pathogens from the culture of the specimens subsequently collected from the original site.

The empirical antimicrobial regimen (i.e., that used before in-vitro susceptibility data were available) was classified as inadequate when it did not include any agent displaying in-vitro activity against the isolated pathogen.

Clinical outcomes were independently evaluated by two physicians (GDP and GM) who were blinded to the type of treatment. When judgments were discordant (about 3\% of patients), the reviewers reassessed the data and reached a consensus decision.

\section{Microbiological analysis}

Strains were identified to the species level with matrixassisted laser desorption ionization-time-of-flight (MALDITOF) mass spectrometry (MS) (BrukerDaltonik or Vitek MS; bioMérieux, Marcy l'Etoile, France). The antibiotic susceptibility profiling of isolates was carried out with the Vitek 2 system (bioMérieux). Results were interpreted according to the European Committee on Antimicrobial Susceptibility Testing (EUCAST) clinical breakpoints. Minimum inhibitory concentrations (MICs) of colistin and tigecycline were also assessed with the Sensititre broth microdilution method (Trek Diagnostic Systems, Cleveland, $\mathrm{OH}, \mathrm{USA})$. The phenotypic detection of carbapenemase expression in isolates with MICs $>1 \mathrm{mg} / \mathrm{L}$ for carbapenems was carried out according to EUCAST guidelines. Isolates screened as positive were further investigated by polymerase chain reaction to identify genes for class A carbapenemases (KPC and GES enzymes), class B metallo- $\beta$ lactamases (VIM, IMP, and NDM enzymes), and class D carbapenemases (OXA-23, OXA-24/40, OXA-48, OXA-51, OXA-55, OXA-58, and OXA-143) [32].

\section{Statistical analysis}

The Kolmogorov-Smirnov test was used to evaluate the distribution of variables. Data with a nonnormal distribution were assessed with the Mann-Whitney test, and the median and selected centile (25th-75th) values are given. The data with a normal distribution were assessed with the Student's $t$ test. Categorical variables are given as proportions, and were analyzed with the chi-square test or Fisher's exact test, as appropriate. $p<0.05$ was considered significant. The crude odds ratio (OR) and 95\% CI were calculated for each variable. We included all variables in the multivariable logistic regression if they reached $p \leq 0.1$ on univariate analysis. A stepwise selection procedure was used to select variables for inclusion in the final model. The Hosmer-Lemeshow goodness-of-fit test and receiver operating characteristic (ROC) curve analysis were used to assess the goodness of the logistic final model. The Kaplan-Meier method was used for the survival analysis. All statistical analyses were performed using SAS 9.4 software (SAS Institute Inc, Cary, NC, USA).

\section{Results}

\section{Population characteristics and treatment}

During the study period, 243 patients with CR-Kp infection were identified. Among 72 potentially eligible case patients receiving a DC regimen including ertapenem, alone or in combination with another "in vitro active antibiotic" (mainly colistin), 24 patients were excluded (mainly because they were treated for less than 72 hours) and 48 patients were selected for the analysis (Additional file 1: Figure S1). These patients were compared with 96 corresponding controls who received an ST regimen not including carbapenems, matched for SAPS II and SOFA scores (baseline characteristics presented in Table 1).

There were no significant between-cohort differences in terms of demographics, type of admission, presenting features, main comorbidities, and infection types. Duration of ICU stay and the use of mechanical ventilation and vasopressors before infection occurrence were similar in both groups. About $50 \%$ of the patients were affected with CR-Kp pneumonia: 51 patients were classified as VAP, and 23 patients as hospital-acquired pneumonia (HAP) requiring ICU admission due to respiratory failure. Septic shock upon occurrence of infection, along with secondary bacteremia and high procalcitonin levels, were significantly more frequent in patients receiving DC treatment. The overall duration of therapy was significantly longer in the DC group than in controls (17 days vs 11.5 days, $p<0.01)$. Seven patients $(4.9 \%)$ had multiple site infections, and the overall rate of initial inappropriate antibiotic therapy was less than $50 \%$ in both groups. Adjunctive nebulized colistin (2 MUI every 8 hours, via jet/ultrasonic nebulizers) was used in 32 patients with VAP (seven cases and 25 controls). No between-group differences were identified in terms of MV and vasopressor duration after infection. No adverse events potentially associated with the use of the DC regimen were observed in both centers.

In 23 patients $(16 \%)$ the infection was polymicrobial (15 CR Acinetobacter baumannii isolates and eight CR Pseudomonas aeruginosa isolates), without statistically significant differences between the two groups. The rate of combination therapy including at least one other active antibiotic was higher in the DC group $(72.9 \%$ vs $54.2 \%, p=0.05)$ : antibiotic association details for the DC and ST groups are presented in Table 2.

More than $90 \%$ of detected $K p$ produced class A carbapenemases (KPC), while the rest of the isolates harbored genes for class $B$ metallo- $\beta$-lactamases and class $D$ carbapenemases (OXA-48). About $40 \%$ of the isolated CR- $K p$ was resistant to either colistin or tigecycline (colistin sensitivity: $58.3 \%$ in the DC group vs $66.7 \%$ in controls, $p=0.42$; 
Table 1 Demographic and clinical characteristics of the 144 patients with KPC Kp invasive infection included in the study

\begin{tabular}{|c|c|c|c|}
\hline \multirow[t]{2}{*}{ Variable } & \multicolumn{2}{|c|}{ Number (\%) of patients } & \multirow[t]{2}{*}{$p$ value } \\
\hline & $\overline{D C}$ group $(n=48)$ & ST group $(n=96)$ & \\
\hline \multicolumn{4}{|l|}{ Demographics and comorbidities } \\
\hline Age (years), mean $\pm S D$ & $55.5 \pm 15$ & $61.3 \pm 12$ & 0.06 \\
\hline Males & $35(72.9)$ & $58(60.4)$ & 0.19 \\
\hline $\mathrm{CHF}$ & $18(37.5)$ & $27(28.1)$ & 0.34 \\
\hline COPD & $11(22.9)$ & $12(12.5)$ & 0.17 \\
\hline CRF & $8(16.7)$ & $7(7.3)$ & 0.15 \\
\hline Diabetes & $17(35.4)$ & $31(32.3)$ & 0.85 \\
\hline CLD & $10(20.8)$ & $8(8.3)$ & 0.06 \\
\hline Neoplasm & $2(4.2)$ & $12(12.5)$ & 0.21 \\
\hline Immunosuppressive status & $19(39.6)$ & $26(27.1)$ & 0.18 \\
\hline ICU stay before infection (days), median (IQR) & $11(4-24)$ & $7(1-22.5)$ & 0.08 \\
\hline Duration of MV before infection (days), median (IQR) & $6(1.5-15.5)$ & $5(1-14)$ & 0.57 \\
\hline Duration of vasopressors before infection (days), median (IQR) & $3(1-10)$ & $1.5(0-6)$ & 0.11 \\
\hline \multicolumn{4}{|l|}{ Presenting feature } \\
\hline Medical admission & $25(52.1)$ & $59(61.5)$ & 0.37 \\
\hline Surgical admission & $18(37.5)$ & $30(31.3)$ & 0.28 \\
\hline Trauma admission & $5(10.4)$ & $11(11.5)$ & 0.92 \\
\hline SAPS II score, median (IQR) & $44(36-56)$ & $46(36-57)$ & 0.56 \\
\hline SOFA score at infection, median (IQR) & $9(7-11)$ & $8(6-10)$ & 0.33 \\
\hline Septic shock on occurrence of infection & $36(75)$ & $43(44.8)$ & $<0.01$ \\
\hline ARDS on occurrence of infection & $18(37.5)$ & $23(23.9)$ & 0.13 \\
\hline CRRT on occurrence of infection & $20(41.7)$ & $26(27.1)$ & 0.13 \\
\hline PCT on occurrence of infection (ng/ml), median (interval) & $6.1(3.2-50.4)$ & $3.1(0.8-5.9)$ & $<0.01$ \\
\hline \multicolumn{4}{|l|}{ Type of infection } \\
\hline Pneumonia & $25(52.1)$ & $49(51)$ & 0.95 \\
\hline$|A|$ & $9(18.7)$ & $10(10.4)$ & 0.19 \\
\hline SSTI & $1(2.1)$ & $11(11.5)$ & 0.06 \\
\hline UTI & $3(6.2)$ & $9(9.3)$ & 0.75 \\
\hline CVC BSI & $8(16.7)$ & $10(10.4)$ & 0.42 \\
\hline Primary BSI & $6(12.5)$ & $10(10.4)$ & 0.92 \\
\hline Secondary BSI & $23(47.9)$ & $26(27.1)$ & 0.02 \\
\hline Multiple site infection & $4(8.2)$ & $3(3.1)$ & 0.54 \\
\hline \multicolumn{4}{|l|}{ Therapeutic aspects } \\
\hline ॥AT & $13(27.1)$ & $40(41.7)$ & 0.16 \\
\hline Overall duration of treatment (days), median (interval) & $17(11.5-25.5)$ & $11.5(7.5-15.5)$ & $<0.01$ \\
\hline \multicolumn{4}{|l|}{ Clinical and microbiological outcome } \\
\hline 28-day mortality & $14(29.2)$ & $46(47.9)$ & 0.04 \\
\hline 90-day mortality & $24(50)$ & $58(60.4)$ & 0.31 \\
\hline Clinical cure & $30(62.5)$ & $47(48.9)$ & 0.17 \\
\hline Microbiological eradication ${ }^{a}$ & $22(50)$ & $31(38.3)$ & 0.27 \\
\hline Duration of MV after infection (days), median (interval) & $14(7.5-31)$ & $11.5(7-19)$ & 0.16 \\
\hline Duration of vasopressors after infection (days), median (interval) & $10.5(3.5-27.5)$ & $8(4.5-12)$ & 0.19 \\
\hline
\end{tabular}

Data presented as $N(\%)$, unless otherwise indicated. Bold data are significant

IQR interquartile range, KPC Klebsiella pneumoniae carbapenemase, Kp Klebsiella pneumoniae, DC double carbapenem, ST standard treatment, SAPS Simplified Acute Physiology Score, SOFA Sequential Organ Failure Assessment, ICU intensive care unit, MV mechanical ventilation, ARDS acute respiratory distress syndrome, CRRT continuous renal replacement therapy, $P C T$ procalcitonin, CHF chronic heart failure, COPD chronic obstructive pulmonary disease, CRF chronic renal failure, CLD chronic liver disease, IAI intra-abdominal infection, SSTI skin and soft tissue infection, UTI urinary tract infection, CVC central vascular catheter, $B S I$ bloodstream infection, IIAT initial inappropriate antimicrobial therapy

${ }^{a}$ Microbiological outcome was analyzed in 125 patients: 44 patients (DC group) and 81 patients (ST group) 
Table 2 Therapeutic and microbiological details according to the type of treatment

\begin{tabular}{|c|c|c|c|}
\hline \multirow[t]{2}{*}{ Variable } & \multicolumn{2}{|c|}{ Number (\%) of patients } & \multirow[t]{2}{*}{$p$ value } \\
\hline & DC group $(n=48)$ & ST group $(n=96)$ & \\
\hline Polymicrobial infection & $6(12.5)$ & $17(17.7)$ & 0.57 \\
\hline CR Acinetobacter baumannii & $3(6.25)$ & $12(12.5)$ & 0.4 \\
\hline CR Pseudomonas aeruginosa & $3(6.25)$ & $5(5.2)$ & 0.89 \\
\hline Combined targeted therapy & $35(72.9)$ & $52(54.2)$ & 0.05 \\
\hline DC + colistin & 19 (39.6) & - & - \\
\hline $\mathrm{DC}+$ gentamicin & $8(16.9)$ & - & - \\
\hline DC + tigecycline & $3(6.25)$ & - & - \\
\hline DC + colistin + tigecycline & $2(4.2)$ & - & - \\
\hline $\mathrm{DC}+$ colistin + gentamicin & $3(6.25)$ & - & - \\
\hline Colistin + tigecycline & - & $22(22.9)$ & - \\
\hline Colistin + gentamicin & - & $13(13.5)$ & - \\
\hline Gentamicin + tigecycline & - & $10(10.4)$ & - \\
\hline Colistin + tigecycline + gentamicin & - & $7(7.3)$ & - \\
\hline Extensively drug-resistant strains ${ }^{a}$ & $32(66.7)$ & $31(32.3)$ & $<0.01$ \\
\hline Colistin MIC $\leq 2 \mu \mathrm{g} / \mathrm{ml}$ & $28(58.3)$ & $64(66.7)$ & 0.42 \\
\hline Gentamicin MIC $\leq 2 \mu \mathrm{g} / \mathrm{ml}$ & $15(31.3)$ & $72(75)$ & $<0.01$ \\
\hline Tigecycline $\mathrm{MIC} \leq 1 \mu \mathrm{g} / \mathrm{ml}^{\mathrm{b}}$ & $16(47.1)$ & $58(60.4)$ & 0.27 \\
\hline Suboptimal MIC values ${ }^{c}$ & $10(20.8)$ & $14(14.6)$ & 0.5 \\
\hline
\end{tabular}

Bold data are significant

DC double carbapenem, ST standard treatment, $C R$ carbapenem resistant, MIC minimal inhibitory concentration

${ }^{a}$ Strains resistant to at least one agent in all but two or fewer usually active antimicrobial categories

${ }^{\mathrm{b}} \mathrm{MIC}$ values were available in 130 patients: 34 pts (DC group) and 96 patients (ST group)

'Gentamicin $(4 \mu \mathrm{g} / \mathrm{ml})$, tigecycline $(2 \mu \mathrm{g} / \mathrm{ml})$

tigecycline: $47.1 \%$ in the DC group vs $60.4 \%$ in controls, $p$ $=0.27$ ). Gentamicin sensitivity was significantly lower in patients receiving the ST regimen compared with the DC group $(75 \%$ vs $31.3 \%, p<0.01)$. Furthermore, 24 strains (16.7\%) showed suboptimal MIC values for at least one rescue antimicrobial molecule (pharmacological and microbiological details presented in Table 2).

\section{Outcomes and predictors of mortality}

Twenty-eight-day mortality was significantly higher in patients receiving ST compared with those who were treated with the DC regimen (47.9\% vs $29.2 \%, p=0.04$ ). Despite a trend toward a better outcome in the DC group, the rates of clinical cure and microbiological eradication did not significantly differ between the groups. Overall, considering the 90 -day mortality after infection, $50 \%$ of patients in the DC group were alive compared with $39.6 \%$ in controls, but despite such a reduction trend the difference was nonsignificant (Table 1).

However, stratifying the population according to the pattern of microbiological resistance, we found that clinical cure and microbiological eradication associated with DC were significantly higher in patients infected with CR- $K p$ resistant to colistin (clinical cure: 13/20 (65\%) vs 10/32 (31.3\%), $p=0.03$; microbiological eradication: 11/19 (57.9\%) vs 7/27 (25.9\%), $p=0.04)$. Clinical cure was also higher for strains resistant to both gentamicin and colistin (clinical cure: $6 / 12(50 \%)$ vs $0 / 6(0), p=0.05$; microbiological eradication: $6 / 11(54.5 \%)$ vs $0 / 5(0), p=0.09)$. No significant between-group differences were found in patients with germs resistant to either gentamicin or tigecycline, or to both gentamicin and tigecycline and to both colistin and tigecycline. Five infections were due to pan-resistant strains (three pneumonia and two complicated intra-abdominal infections): these were treated with the DC regimen, with a $40 \%$ rate of clinical cure and microbiological eradication (2/ 5) (Fig. 1).

On univariate analysis (Table 3), deceased numbers of patients had higher SAPS II and SOFA scores compared with survivors. Acute kidney injury requiring CRRT, polymicrobial infections, and inappropriate initial antimicrobial treatment occurred more frequently in patients who died. In multivariable logistic regression analysis, the DC regimen was the sole independent factor associated with 28-day survival, while higher SAPS II and SOFA score were significantly associated with mortality (Table 4). The multivariate Cox-regression model showed 28-day survival to be associated with the DC regimen (OR 0.43, 95\% CI 0.23-0.79), and the difference in survival between treatment groups was also found on Kaplan-Meier survival curve analysis ( $p$ 

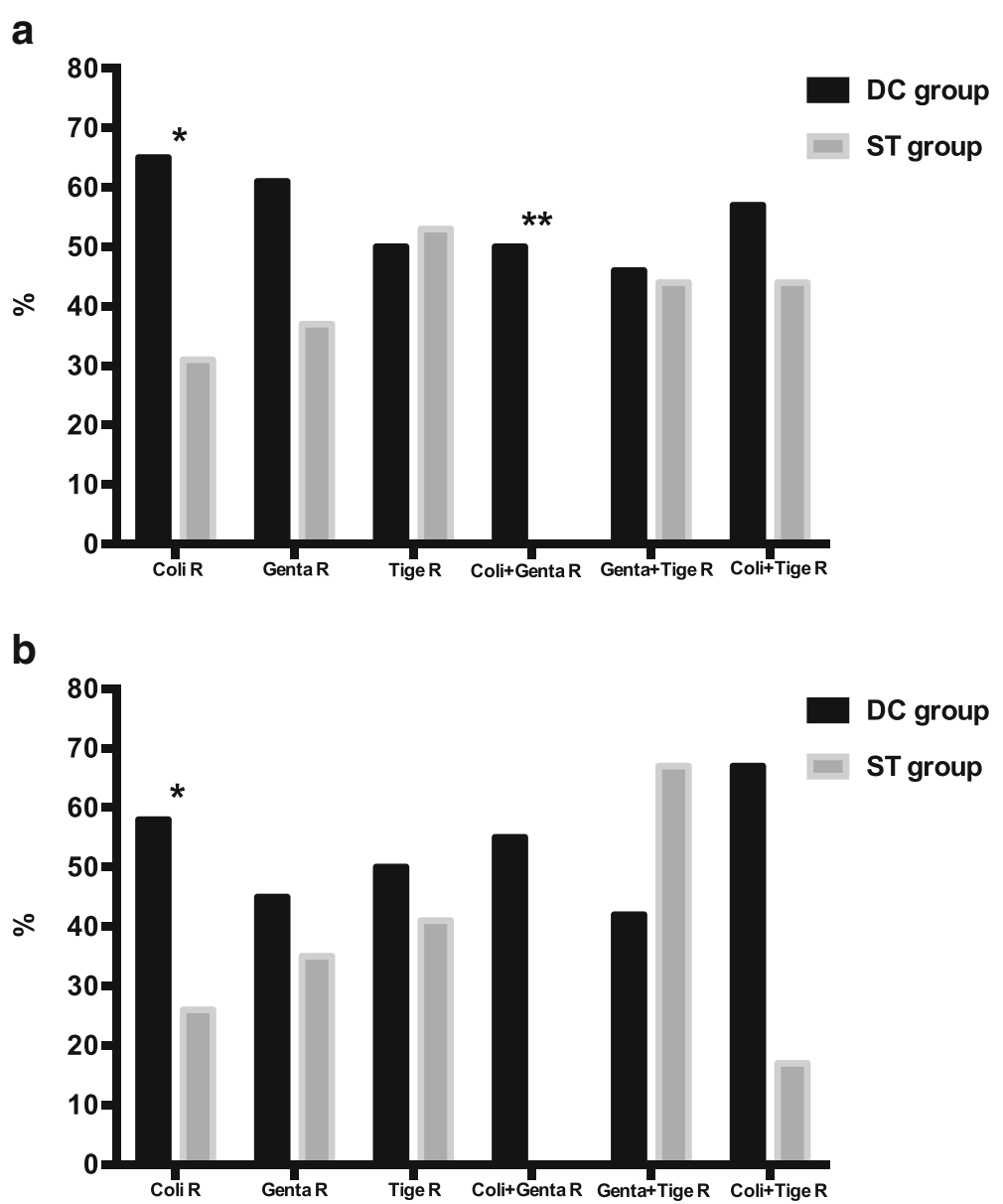

Fig. 1 a Clinical cure rate according to antibiotic resistance. ${ }^{*} p=0.03,{ }^{* *} p=0.05$. b Microbiological eradication rate according to antibiotic resistance. ${ }^{*} p=0.04$. $R$ resistance, $D C$ double carbapenem, ST standard treatment, Coli colistin, Genta gentamicin, Tige tigecycline

$=0.02)$. Unfortunately, such survival benefit was not maintained at 90-day mortality $(p=0.08)$ (Fig. 2).

\section{Discussion}

In this population of critically ill patients affected with severe invasive infections due to carbapenem-resistant Klebsiella pneumoniae, the use of a DC regimen including ertapenem reduced 28-day mortality. Furthermore, clinical cure and microbiological eradication were more frequent in patients treated with the DC for colistinresistant $\mathrm{CR}-\mathrm{Kp}$ infections. Our findings support recent data suggesting the clinical efficacy of a DC regimen for the treatment of CR-Kp infections [21, 33].

$K p$-producing carbapenemases are resistant to all betalactams, and to most aminoglycosides, fluoroquinolones, and sulphonamides [9]. The few remaining options are colistin, tigecycline, fosfomycin, and gentamicin, which usually harbor high MIC values, and share a suboptimal pharmacokinetic profile in terms of distribution at many infection sites $[7,13,34]$. Nonetheless, the use of gentamicin and colistin is frequently limited due to the risk of renal toxicity, and experience with the novel beta-lactam/ beta-lactamase inhibitor combinations (e.g., ceftazidimeavibactam) in this setting is exiguous [16]. The rationale behind the usefulness of a DC regimen is intriguing: ertapenem, binding with high affinity to the active site of $K p$ carbapenemase, would be able to prevent the hydrolysis of the coadministered drug (i.e., meropenem, imipenem, or doripenem), which may preserve its bactericidal activity against the infecting isolate [35]. Indeed, carbapenemase consumption by ertapenem means that a high concentration of the associated drug can be active in the infection site, where a lower amount of carbapenemases will be available to degrade the administered antibiotic.

The preliminary observation of DC efficacy by Bulik and Nicolau in 2011 [18] was confirmed by clinical experiences documenting the efficacy of the DC regimen, including ertapenem plus meropenem or doripenem, in patients treated for CR-Kp infections [19-21, 33, 36, 37]. Recently a large series by Souli et al. [19] reported encouraging data on the use of a DC regimen as the exclusive therapy for 
Table 3 Univariate analysis of factors associated with 28-day mortality

\begin{tabular}{|c|c|c|c|c|}
\hline \multirow[t]{2}{*}{ Variable } & \multicolumn{2}{|c|}{ Number (\%) of patients } & \multicolumn{2}{|c|}{$\underline{\text { Univariate analysis }}$} \\
\hline & Alive $(n=84)$ & Deceased $(n=60)$ & $p$ value & OR $(95 \% \mathrm{Cl})$ \\
\hline \multicolumn{5}{|l|}{ Demographics and comorbidities } \\
\hline Age (years) & $57.7 \pm 15$ & $61.7 \pm 10$ & 0.08 & $1.02(0.99-1.05)$ \\
\hline Males & $58(69.1)$ & $35(58.3)$ & 0.19 & $0.63(0.31-1.25)$ \\
\hline $\mathrm{CHF}$ & $24(28.6)$ & $21(35)$ & 0.41 & $1.34(0.66-2.74)$ \\
\hline COPD & $14(16.7)$ & $9(15)$ & 0.79 & $0.88(0.35-2.2)$ \\
\hline CRF & $8(9.5)$ & $7(11.7)$ & 0.68 & $1.25(0.43-3.67)$ \\
\hline Diabetes & $25(29.8)$ & $23(38.3)$ & 0.28 & $1.47(0.73-2.95)$ \\
\hline CLD & $8(9.5)$ & $10(16.7)$ & 0.21 & $1.9(0.7-5.14)$ \\
\hline Neoplasm & $7(8.3)$ & $7(11.7)$ & 0.51 & $1.48(0.48-4.38)$ \\
\hline Immunosuppressive status & $27(32.1)$ & $18(30)$ & 0.78 & $0.9(0.44-1.85)$ \\
\hline ICU stay before infection (days), median (IQR) & $8.5(1-21)$ & $8(1-23)$ & 0.69 & $1(0.98-1.01)$ \\
\hline Duration of MV before infection (days), median (IQR) & $6(1-13.5)$ & $5.5(0.5-15)$ & 0.79 & $1.01(0.98-1.03)$ \\
\hline Duration of vasopressors before infection (days), median (IQR) & $2(0-7.5)$ & $2(0-7)$ & 0.68 & $0.99(0.97-1.02)$ \\
\hline \multicolumn{5}{|l|}{ Presenting features, type of infection, and therapy } \\
\hline Medical admission & $45(53.6)$ & $39(65)$ & 0.17 & $1.61(0.81-3.18)$ \\
\hline Surgical admission & $29(34.5)$ & $15(25)$ & 0.22 & $0.63(0.3-1.32)$ \\
\hline Trauma admission & $10(11.9)$ & $6(10)$ & 0.72 & $0.82(0.28-2.4)$ \\
\hline SAPS II score, median (IQR) & $39(34-50)$ & $55(45.5-63)$ & $<0.01$ & $1.07(1.04-1.08)$ \\
\hline SOFA score on occurrence of infection, median (IQR) & $8(5-9)$ & $9(8-11)$ & $<0.01$ & $1.36(1.17-1.57)$ \\
\hline SS on occurrence of infection & $42(50)$ & $37(61.7)$ & 0.17 & $1.6(0.82-3.16)$ \\
\hline ARDS on occurrence of infection & $21(25)$ & $20(33.3)$ & 0.28 & $1.5(0.72-3.11)$ \\
\hline CRRT on occurrence of infection & $22(26.2)$ & $24(40)$ & 0.08 & $1.88(0.92-3.82)$ \\
\hline PCT on occurrence of infection ( $\mathrm{ng} / \mathrm{ml}$ ), median (interval) & $3.4(0.71-7.5)$ & $4.3(2.3-8.6)$ & 0.43 & $1(0.99-1.01)$ \\
\hline \multicolumn{5}{|l|}{ Type of infection and therapy } \\
\hline Pneumonia & $41(48.8)$ & $33(55)$ & 0.46 & $1.28(0.66-2.49)$ \\
\hline$|A|$ & $14(16.7)$ & $5(8.3)$ & 0.15 & $0.45(0.15-1.34)$ \\
\hline SSTI & $8(9.5)$ & $4(6.7)$ & 0.55 & $0.67(0.19-2.37)$ \\
\hline UTI & $6(7.1)$ & $6(10)$ & 0.54 & $1.44(0.44-4.72)$ \\
\hline CVC BSI & $11(13.1)$ & $7(11.7)$ & 0.8 & $0.88(0.32-2.41)$ \\
\hline Primary BSI & $9(10.7)$ & $7(11.7)$ & 0.86 & $1.1(0.39-3.14)$ \\
\hline Secondary BSI & $30(35.7)$ & $19(31.7)$ & 0.61 & $0.83(0.41-1.69)$ \\
\hline Polymicrobial infection & $8(9.5)$ & $15(25)$ & 0.01 & $3.17(1.25-8.06)$ \\
\hline Double carbapenem therapy & $34(40.5)$ & $14(23.3)$ & 0.03 & $0.45(0.21-0.94)$ \\
\hline IIAT & $26(30.9)$ & $27(45)$ & 0.08 & $1.82(0.92-3.63)$ \\
\hline Combination targeted therapy & $48(57.1)$ & $39(65)$ & 0.34 & $1.39(0.7-2.76)$ \\
\hline Duration of active treatment (days), median (IQR) & $14(8-18.5)$ & $10(8-14.5$ & 0.13 & $0.96(0.93-1.01)$ \\
\hline
\end{tabular}

Data presented as $N(\%)$, unless otherwise indicated. Bold data are significant

OR odds ratio, IQR interquartile range, SAPS Simplified Acute Physiology Score, SOFA Sequential Organ Failure Assessment, ICU intensive care unit, MV mechanical ventilation, SS septic shock, ARDS acute respiratory distress syndrome, CRRT continuous renal replacement therapy, $P C T$ procalcitonin, CHF chronic heart failure, COPD chronic obstructive pulmonary disease, CRF chronic renal failure, CLD chronic liver disease, IAI intra-abdominal infection, SSTI skin and soft tissue infection, UTI urinary tract infection, CVC central vascular catheter, BSI bloodstream infection, IIAT initial inappropriate antimicrobial therapy

extensively or pan-drug-resistant CR-Kp, further summarizing the actual clinical evidence in the field. In their cohort, a relevant clinical success was reached $(77.8 \%)$, and also in the pan-resistant group the clinical and microbiological success was comparable (78.5\%). In their review of the literature, a trend toward DC efficacy was highlighted, although there were some discrepancies among series. 
Table 4 Multivariable logistic regression analysis of factors associated with 28-day mortality

\begin{tabular}{lll}
\hline Variable & $p$ value & OR (95\% Cl) \\
\hline SAPS II score & $<0.01$ & $1.08(1.04-1.23)$ \\
SOFA score & $\leq 0.01$ & $1.36(1.33-1.63)$ \\
Double carbapenem treatment & 0.02 & $0.33(0.13-0.87)$
\end{tabular}

We included all variables in the multivariable logistic regression if they reached $p \leq 0.1$ on univariate analysis. A stepwise selection procedure was used to select variables for inclusion in the final model

ROC curve analysis was used to assess the goodness of the final logistic regression model (AUC $\pm \mathrm{SE}=0.85 \pm 0.034$ with $95 \% \mathrm{Cl} 0.78-0.91$; chi-square statistics $p<0.001)$

$A U C$ area under the curve, $O R$ odds ratio, $R O C$ receiver operating characteristic, SAPS Simplified Acute Physiology Score, SOFA Sequential Organ Failure Assessment, SE standard error

In our study, we aimed at enhancing such evidence by comparing a larger cohort of DC-treated patients with a matched cohort of similar patients.

Given residual antimicrobial susceptibility, more than half of our patients receiving DC (35 [72.9\%]) were treated with another active drug, mainly colistin. This approach is reasonable, and recent data suggest that the coadministration of colistin may increase the in-vivo and in-vitro activity of DC, and should be preferred in critically ill patients where an early clinical response is warranted [33, 38]. Similarly, in line with expert opinion, 52 of 96 controls were managed with a combined targeted

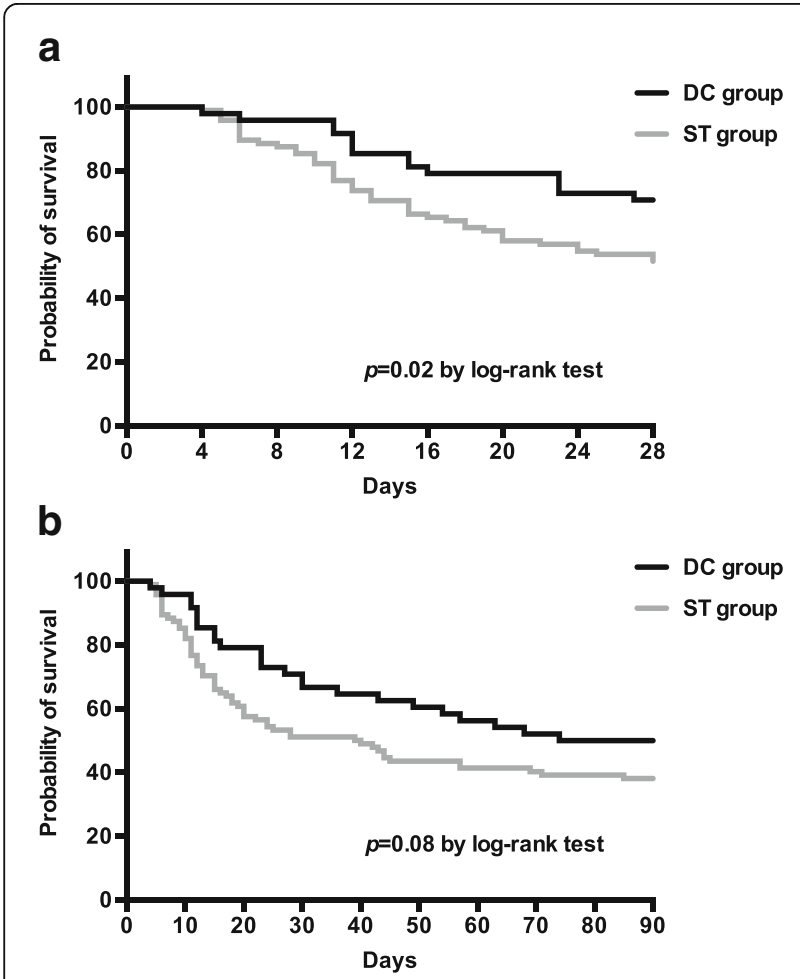

Fig. 2 Kaplan-Meier curves showing the impact of DC therapy (black line) versus ST (gray line) on a 28-day mortality and $\mathbf{b}$ 90-day mortality. DC double carbapenem, ST standard treatment therapy [39-41]. A carbapenem-sparing regimen has been proposed by Sbrana et al. [39], observing that the administration of tigecycline plus gentamicin or colistin was effective for the treatment of $24 \mathrm{CR}-K p$ infections in trauma patients, with a $14 \%$ mortality rate, further suggesting the usefulness of this approach when tigecycline is administered at a higher than standard dose (i.e., $50 \mathrm{mg}$ every 12 hours after a $100 \mathrm{mg}$ loading dose) [42]. On the other hand, recent data advocate the efficacy of combining high dosages of meropenem with colistin or tigecycline for the treatment of Acinetobacter infections [41]. These antibiotic associations have been seen to reduce mortality in patients with life-threatening infections due to extensively drug-resistant Acinetobacter and Klebsiella, but the benefits of meropenem can be expected only in the presence of MIC values close to susceptibility breakpoints. This kind of effect did not seem to have influenced the outcome of our cohort treated with DC because all of the strains were resistant to meropenem. In any event, we also have to address the idea of limiting the use of colistin in settings where KPC infections are frequent, given the alarming increase of colistin resistance in high-endemicity areas [43, 44].

Of note, we found a longer duration of antibiotic therapy in the DC group: such a result may be interpreted in light of the clinical profile of the DC group, in which patients, despite a higher percentage of septic shock, bacteremia rate, and PCT levels, showed higher survival together with a longer period of ICU support.

Moreover, the discrepancy in the effect of DC therapy between 28-day and 90-day mortality may be explained by the higher clinical impact of the septic episode on the short-term outcomes, along with the role of potential unmeasured confounders that may have influenced the 90-day mortality.

Our study has several limitations. First, although both centers adopted an electronic medical record, due to the observational nature we cannot exclude that unmeasured confounders may have influenced the strong association between DC use and improved survival. Second, synergistic assays for confirming DC in-vitro efficacy were not done routinely, although $\mathrm{CR}-K p$ strains were studied according to genetic testing. Third, we did not check plasmatic carbapenem concentrations, so we can only assume that by optimizing dosages and administration modalities we achieved bactericidal meropenem levels at the infection site. Fourth, due to the use of the Vitek system, we cannot exclude that a small proportion of strains could display meropenem MIC values closer to the susceptibility breakpoint. Finally, we did not use either fosfomycin or ceftazidime-avibactam [45] because both molecules were not available at our centers during the observation period. 
Nonetheless, this is the largest observational study in critically ill patients in whom the use of DC was evaluated, and the first investigation in which such a strategy is compared with other rescue treatments (i.e., colistin, tigecycline, gentamicin), after matching for severity of the disease.

\section{Conclusion}

In the daily challenge of managing critically ill patients with CR-Kp infections, therapeutic options are limited. This observational analysis shows that the association of ertapenem plus meropenem provides a survival benefit, particularly when colistin cannot be used. Larger prospective studies are needed to confirm these findings, and to define the role of this unconventional strategy.

\section{Additional files}

Additional file 1: Figure $\mathbf{S 1}$ showing a flow chart of the study inclusion process. CR-Kp carbapenem-resistant Klebsiella pneumoniae, DC double carbapenem, ST standard treatment. (PDF $92.6 \mathrm{~kb}$ )

\section{Abbreviations}

ARDS: Acute respiratory distress syndrome; BSI: Bloodstream infection; CRKp: Carbapenem-resistant Klebsiella pneumoniae; CRRT: Continuous renal replacement therapy; DC: Double carbapenem; EUCAST: European Committee on Antimicrobial Susceptibility Testing; HAP: Hospital-acquired pneumonia; IAl: Intraabdominal infection; ICU: Intensive care unit; MALDITOF: Matrix-assisted laser desorption ionization-time-of-flight; MIC: Minimum inhibitory concentration; MS: Mass spectrometry; OR: Odds ratio; ROC: Receiver operating characteristic; SAPS: Simplified Acute Physiology Score; SOFA: Sequential Organ Failure Assessment; SS: Septic shock; SSTI: Skin and soft tissue infection; ST: Standard treatment; TDM: Therapeutic drug monitoring; UTI: Urinary tract infection; VAP: Ventilator-associated pneumonia

\section{Acknowledgements}

The authors are indebted to Warren Blumberg, science editor at ISMETT, for his help in revising the manuscript.

\section{Funding}

The study did not receive any funding.

\section{Availability of data and materials}

The datasets used and/or analyzed during the current study are available from the corresponding author on reasonable request.

\section{Authors' contributions \\ GDP and GM had full access to all data in the study and take responsibility for the integrity and the accuracy of the data analysis; they contributed equally to the manuscript. GDP, MA, GM, and AA conceived the study, participated in study design and coordination, and helped to draft the manuscript. LM and GDP were in charge of the statistical analysis, participated in analysis and interpretation of data, helped to draft the manuscript, and critically revised the manuscript for important intellectual content. GM, MSV, SLC, and VDG collected data for the study and participated in statistical analysis. GP, DDC, RDS, GC, PP, LM, and TS participated in the conception, design, and development of the database, helped in analysis and interpretation of data, helped draft the manuscript, and critically revised the manuscript for important intellectual content. All authors read and approved the final manuscript.}

\section{Ethics approval and consent to participate}

The study was approved by the local ethics committees (approval numbers: Comitato Etico UCSC 14669/15; Comitato Etico Palermo $2 \mathrm{n}^{\circ}$ 359/2015). Because of its observational, noninterventional design, informed consent was waived.
Consent for publication

Not applicable.

\section{Competing interests}

The authors declare that they have no competing interests.

\section{Publisher's Note}

Springer Nature remains neutral with regard to jurisdictional claims in published maps and institutional affiliations.

\section{Author details}

${ }^{1}$ Department of Anesthesiology and Intensive Care, Università Cattolica del Sacro Cuore, Fondazione Policlinico Agostino Gemelli, Rome, Italy. ${ }^{2}$ Department of Anesthesia and Intensive Care, IRCCS-ISMETT (Istituto Mediterraneo per i Trapianti e Terapie ad alta specializzazione), Palermo, Italy. ${ }^{3}$ Department of Laboratory Medicine and Advanced Biotechnologies, IRCCS-ISMETT (Istituto Mediterraneo per i Trapianti e Terapie ad alta specializzazione), Palermo, Italy. ${ }^{4}$ Clinical Pharmacy, IRCCS-ISMETT (Istituto Mediterraneo per i Trapianti e Terapie ad alta specializzazione), Palermo, Italy. ${ }^{5}$ Institute of Microbiology, Università Cattolica del Sacro Cuore, Fondazione Policlinico Agostino Gemelli, Rome, Italy. ${ }^{6}$ Fondazione Policlinico A. Gemelli. Università Cattolica del Sacro Cuore, Largo A. Gemelli 8, 00168 Rome, Italy.

Received: 6 April 2017 Accepted: 20 June 2017

Published online: 05 July 2017

\section{References}

1. Tumbarello M, Trecarichi EM, Tumietto F, Del Bono V, De Rosa FG, Bassetti M, Losito AR, Tedeschi S, Saffioti C, Corcione S, et al. Predictive models for identification of hospitalized patients harboring KPC-producing Klebsiella pneumoniae. Antimicrob Agents Chemother. 2014;58(6):3514-20.

2. Baraniak A, Izdebski R, Fiett J, Herda M, Derde LP, Bonten MJ, Adler A, Carmeli Y, Goossens H, Hryniewicz W, et al. KPC-Like Carbapenemaseproducing enterobacteriaceae colonizing patients in Europe and Israel. Antimicrob Agents Chemother. 2015;60(3):1912-7.

3. Albiger B, Glasner C, Struelens MJ, Grundmann H, Monnet DL. Carbapenemaseproducing Enterobacteriaceae in Europe: assessment by national experts from 38 countries, May 2015. Euro Surveill. 2015;20(45):1-18. doi:10.2807/1560-7917.ES. 2015.20.45.30062

4. Dautzenberg MJ, Wekesa AN, Gniadkowski M, Antoniadou A, Giamarellou H, Petrikkos GL, Skiada A, Brun-Buisson C, Bonten MJ, Derde LP. The association between colonization with carbapenemaseproducing enterobacteriaceae and overall ICU mortality: an observational cohort study. Crit Care Med. 2015;43(6):1170-7.

5. Gutierrez-Gutierrez B, Salamanca E, de Cueto M, Hsueh PR, Viale P, Pano-Pardo JR, Venditti M, Tumbarello M, Daikos G, Pintado V, et al. A predictive model of mortality in patients with bloodstream infections due to carbapenemase-producing enterobacteriaceae. Mayo Clin Proc. 2016;91(10):1362-71.

6. Karaiskos I, Giamarellou H. Multidrug-resistant and extensively drug-resistant Gram-negative pathogens: current and emerging therapeutic approaches. Expert Opin Pharmacother. 2014;15(10):1351-70.

7. Poulakou G, Matthaiou DK, Bassetti M, Erdem H, Dimopoulos G, Curcio DJ, Carlet J, Lipman J, Timsit JF, Giamarellou H et al. "Salvage treatment" for infections by extensively- and pan-drug-resistant pathogens is common and often sub-optimal. Intensive Care Med. 2017. doi:10.1007/s00134-017-4796y. [Epub ahead of print]

8. Decker B, Masur H. Bad bugs, no drugs: are we part of the problem, or leaders in developing solutions? Crit Care Med. 2015;43(6):1153-5.

9. Bassetti M, De Waele JJ, Eggimann P, Garnacho-Montero J, Kahlmeter G, Menichetti F, Nicolau DP, Paiva JA, Tumbarello M, Welte T, et al. Preventive and therapeutic strategies in critically ill patients with highly resistant bacteria. Intensive Care Med. 2015;41(5):776-95.

10. Munoz-Price LS, Rosa R, Castro JG, Laowansiri P, Latibeaudiere R, Namias N, Tarima S. Evaluating the impact of antibiotic exposures as time-dependent variables on the acquisition of carbapenem-resistant Acinetobacter baumannii. Crit Care Med. 2016:44(10):e949-56.

11. Tumbarello M, Trecarichi EM, De Rosa FG, Giannella M, Giacobbe DR, Bassetti M, Losito AR, Bartoletti M, Del Bono V, Corcione S, et al. Infections caused by KPC-producing Klebsiella pneumoniae: differences 
in therapy and mortality in a multicentre study. J Antimicrob Chemother. 2015;70(7):2133-43.

12. Tumbarello M, Viale P, Bassetti M, De Rosa FG, Spanu T, Viscoli C. Infections caused by KPC-producing Klebsiella pneumoniae: differences in therapy and mortality in a multicentre study_authors' response. J Antimicrob Chemother. 2015;70(10):2922

13. Gutierrez-Gutierrez B, Salamanca E, de Cueto M, Hsueh PR, Viale P, PanoPardo JR, Venditti M, Tumbarello M, Daikos G, Canton R et al. Effect of appropriate combination therapy on mortality of patients with bloodstream infections due to carbapenemase-producing Enterobacteriaceae (INCREMENT): a retrospective cohort study. Lancet Infect Dis. 2017;17:726-34. doi:10.1016/S1473-3099(17)30228-1.

14. Tzouvelekis LS, Markogiannakis A, Piperaki E, Souli M, Daikos GL. Treating infections caused by carbapenemase-producing Enterobacteriaceae. Clin Microbiol Infect. 2014;20(9):862-72.

15. Zusman O, Altunin S, Koppel F, Dishon Benattar Y, Gedik H, Paul M. Polymyxin monotherapy or in combination against carbapenem-resistant bacteria: systematic review and meta-analysis. J Antimicrob Chemother. 2017;72(1):29-39.

16. Shields RK, Potoski BA, Haidar G, Hao B, Doi Y, Chen L, Press EG, Kreiswirth BN, Clancy CJ, Nguyen MH. Clinical outcomes, drug toxicity, and emergence of ceftazidime-avibactam resistance among patients treated for carbapenemresistant enterobacteriaceae infections. Clin Infect Dis. 2016;63(12):1615-8.

17. Oliva A, Gizzi F, Mascellino MT, Cipolla A, D’Abramo A, D'Agostino C, Trinchieri $V$, Russo G, Tierno F, lannetta M, et al. Bactericidal and synergistic activity of double-carbapenem regimen for infections caused by carbapenemaseproducing Klebsiella pneumoniae. Clin Microbiol Infect. 2016;22(2):147-53.

18. Bulik CC, Nicolau DP. Double-carbapenem therapy for carbapenemaseproducing Klebsiella pneumoniae. Antimicrob Agents Chemother. 2011; 55(6):3002-4.

19. Souli M, Karaiskos I, Masgala A, Galani L, Barmpouti E, Giamarellou H. Double-carbapenem combination as salvage therapy for untreatable infections by KPC-2-producing Klebsiella pneumoniae. Eur J Clin Microbiol Infect Dis. 2017. doi:10.1007/s10096-017-2936-5. [Epub ahead of print]

20. Giamarellou H, Galani L, Baziaka F, Karaiskos I. Effectiveness of a doublecarbapenem regimen for infections in humans due to carbapenemaseproducing pandrug-resistant Klebsiella pneumoniae. Antimicrob Agents Chemother. 2013;57(5):2388-90.

21. Cprek JB, Gallagher JC. Ertapenem-containing double-carbapenem therapy for treatment of infections caused by carbapenem-resistant Klebsiella pneumoniae. Antimicrob Agents Chemother. 2015;60(1):669-73.

22. Le Gall JR, Lemeshow S, Saulnier F. A new Simplified Acute Physiology Score (SAPS II) based on a European/North American multicenter study. JAMA. 1993;270(24):2957-63.

23. Ferreira FL, Bota DP, Bross A, Melot C, Vincent JL. Serial evaluation of the SOFA score to predict outcome in critically ill patients. JAMA. 2001;286(14):1754-8.

24. van Zanten AR, van der Meer YG. Prolonged infusion of carbapenems in critically ill patients: time to extend our practice. Crit Care Med. 2013;41(2):676-7.

25. File Jr TM. Recommendations for treatment of hospital-acquired and ventilator-associated pneumonia: review of recent international guidelines. Clin Infect Dis. 2010;51 Suppl 1:S42-7.

26. Mermel LA, Allon M, Bouza E, Craven DE, Flynn P, O'Grady NP, Raad II, Rijnders BJ, Sherertz RJ, Warren DK. Clinical practice guidelines for the diagnosis and management of intravascular catheter-related infection: 2009 Update by the Infectious Diseases Society of America. Clin Infect Dis. 2009;49(1):1-45.

27. Solomkin JS, Mazuski JE, Bradley JS, Rodvold KA, Goldstein EJ, Baron EJ, O'Neill PJ, Chow AW, Dellinger EP, Eachempati SR, et al. Diagnosis and management of complicated intra-abdominal infection in adults and children: guidelines by the Surgical Infection Society and the Infectious Diseases Society of America. Clin Infect Dis. 2010;50(2):133-64.

28. Stevens DL, Bisno AL, Chambers HF, Dellinger EP, Goldstein EJ, Gorbach SL, Hirschmann JV, Kaplan SL, Montoya JG, Wade JC. Practice guidelines for the diagnosis and management of skin and soft tissue infections: 2014 update by the Infectious Diseases Society of America. Clin Infect Dis. 2014;59(2):e10-52.

29. Hooton TM, Bradley SF, Cardenas DD, Colgan R, Geerlings SE, Rice JC, Saint S, Schaeffer AJ, Tambayh PA, Tenke P, et al. Diagnosis, prevention, and treatment of catheter-associated urinary tract infection in adults: 2009 International Clinical Practice Guidelines from the Infectious Diseases Society of America. Clin Infect Dis. 2010;50(5):625-63.

30. Dellinger RP, Levy MM, Rhodes A, Annane D, Gerlach H, Opal SM, Sevransky JE, Sprung CL, Douglas IS, Jaeschke R, et al. Surviving Sepsis Campaign: international guidelines for management of severe sepsis and septic shock, 2012. Intensive Care Med. 2013:39(2):165-228.

31. Ranieri VM, Rubenfeld GD, Thompson BT, Ferguson ND, Caldwell E, Fan E, Camporota L, Slutsky AS. Acute respiratory distress syndrome: the Berlin Definition. JAMA. 2012;307(23):2526-33.

32. Rasheed J. Detction and characterization of antimicrobial resistance genes in pathogenic bacteria. In: Murray PRBE, Jorgensen JH, Landry ML, Pfaller MA, editors. Manual of clinical micribiology, vol. 1. 9th ed. Washington, DC: American Society of Microbiology; 2007. p. 1248-67.

33. Oliva A, D'Abramo A, D'Agostino C, lannetta M, Mascellino MT, Gallinelli C, Mastroianni CM, Vullo V. Synergistic activity and effectiveness of a doublecarbapenem regimen in pandrug-resistant Klebsiella pneumoniae bloodstream infections. J Antimicrob Chemother. 2014;69(6):1718-20.

34. Perez F, El Chakhtoura NG, Papp-Wallace KM, Wilson BM, Bonomo RA. Treatment options for infections caused by carbapenem-resistant Enterobacteriaceae: can we apply "precision medicine" to antimicrobial chemotherapy? Expert Opin Pharmacother. 2016;17(6):761-81.

35. Poirel L, Kieffer N, Nordmann P. In vitro evaluation of dual carbapenem combinations against carbapenemase-producing Enterobacteriaceae. J Antimicrob Chemother. 2016;71(1):156-61.

36. Ceccarelli G, Falcone M, Giordano A, Mezzatesta ML, Caio C, Stefani S, Venditti M. Successful ertapenem-doripenem combination treatment of bacteremic ventilator-associated pneumonia due to colistin-resistant KPC-producing Klebsiella pneumoniae. Antimicrob Agents Chemother. 2013;57(6):2900-1.

37. Daikos GL, Tsaousi S, Tzouvelekis LS, Anyfantis I, Psichogiou M, Argyropoulou A, Stefanou I, Sypsa V, Miriagou V, Nepka M, et al. Carbapenemase-producing Klebsiella pneumoniae bloodstream infections: lowering mortality by antibiotic combination schemes and the role of carbapenems. Antimicrob Agents Chemother. 2014;58(4):2322-8.

38. Oliva A, Scorzolini L, Castaldi D, Gizzi F, De Angelis M, Storto M, D’Abramo A, Aloj F, Mascellino MT, Mastroianni CM, et al. Double-carbapenem regimen, alone or in combination with colistin, in the treatment of infections caused by carbapenem-resistant Klebsiella pneumoniae (CR-Kp). J Infect. 2017;74(1):103-6.

39. Sbrana F, Malacarne P, Viaggi B, Costanzo S, Leonetti P, Leonildi A, Casini B, Tascini C, Menichetti F. Carbapenem-sparing antibiotic regimens for infections caused by Klebsiella pneumoniae carbapenemase-producing $\mathrm{K}$. pneumoniae in intensive care unit. Clin Infect Dis. 2013;56(5):697-700.

40. Tumbarello M, Viale P, Viscoli C, Trecarichi EM, Tumietto F, Marchese A, Spanu T, Ambretti S, Ginocchio F, Cristini F, et al. Predictors of mortality in bloodstream infections caused by Klebsiella pneumoniae carbapenemaseproducing K. pneumoniae: importance of combination therapy. Clin Infect Dis. 2012;55(7):943-50.

41. Cheng A, Chuang YC, Sun HY, Sheng WH, Yang CJ, Liao CH, Hsueh PR, Yang $J$, Shen NJ, Wang JT, et al. Excess mortality associated with colistin-tigecycline compared with colistin-carbapenem combination therapy for extensively drug-resistant Acinetobacter baumannii bacteremia: a multicenter prospective observational study. Crit Care Med. 2015;43(6):1194-204.

42. De Pascale G, Montini L, Pennisi M, Bernini V, Maviglia R, Bello G, Spanu T, Tumbarello M, Antonelli M. High dose tigecycline in critically ill patients with severe infections due to multidrug-resistant bacteria. Crit Care. 2014;18(3):R90

43. Tumbarello M, De Pascale G, Trecarichi EM, De Martino S, Bello G, Maviglia $\mathrm{R}$, Spanu T, Antonelli M. Effect of aerosolized colistin as adjunctive treatment on the outcomes of microbiologically documented ventilatorassociated pneumonia caused by colistin-only susceptible gram-negative bacteria. Chest. 2013;144(6):1768-75.

44. Rocco M, Montini L, De Pascale G, Antonelli M. Dose of colistin: a work in progress? Crit Care. 2015;19:65

45. Buehrle DJ, Shields RK, Chen L, Hao B, Press EG, Alkrouk A, Potoski BA Kreiswirth BN, Clancy CJ, Nguyen MH. Evaluation of the in vitro activity of ceftazidime-avibactam and ceftolozane-tazobactam against meropenemresistant Pseudomonas aeruginosa isolates. Antimicrob Agents Chemother. 2016;60(5):3227-31. 